\title{
Phase analysis of gated myocardial perfusion SPECT and cardiac resynchronization therapy: The good, the bad, and the ugly
}

\author{
Erito Marques de Souza Filho, MD, PhD, ${ }^{\mathrm{a}, \mathrm{b}}$ and Claudio Tinoco Mesquita, MD, \\ $\mathrm{PhD}^{\mathrm{c}, \mathrm{d}}$ \\ a Universidade Federal Fluminense, Post-Graduation in Cardiovascular Sciences Program, \\ Niteroi, Brazil \\ b Universidade Federal Rural do Rio de Janeiro, Nova Iguaçu, Brazil \\ c Universidade Federal Fluminense, EBESERH/Hospital Antonio Pedro, Niteroi, Brazil \\ d Hospital Pró-Cardíaco, Rio de Janeiro, Brazil
}

Received Jan 23, 2020; accepted Jan 24, 2020

doi: $10.1007 / \mathrm{s} 12350-020-02056-w$

See related article, pp. 1128-1135

"... the little things are infinitely the most important',

- Arthur Conan Doyle, The Adventures of Sher-

lock Holmes

Diffusion of a new technology is determined by five characteristics: relative advantage, compatibility, complexity, opportunity for a trial, and observability. Relative advantage is the superiority over current methodologies and is considered one of the most important attributes for a new technology success but for practical use, complexity, opportunity for a trial, and observability are very important. ${ }^{1}$ The use of phase analysis from gated-SPECT myocardial perfusion imaging (MPI) for the evaluation of left ventricular mechanical dyssynchrony (LVMD) is completing 15 years since its first description. ${ }^{2}$ The method is countbased and is anchored in the fact that regional maximum counts in myocardium are linearly proportional to myocardial wall thickening based on the partial volume effect. Onset of mechanical contraction of the region (phase angle) is derived from the fitting of first harmonic Fourier curve to the discrete regional maximum counts during the cardiac cycle. ${ }^{2,3}$ The relative advantage of this method over radionuclide ventriculography is that it

Reprint requests: Erito Marques de Souza Filho, MD, PhD, Universidade Federal Fluminense, Post-Graduation in Cardiovascular Sciences Program, Niteroi, Brazil; mederitomarques@gmail.com J Nucl Cardiol 2021;28:1136-9.

$1071-3581 / \$ 34.00$

Copyright (C) 2020 American Society of Nuclear Cardiology. is very reproducible, ${ }^{4}$ does not increase radiation exposure or adds any significant change in acquisition protocols and, most important, myocardial perfusion scintigraphy has the advantage of showing, in a single test, both ventricular perfusion disturbances and the phase analysis, also allowing the discrimination of scar areas in the LV that are inappropriate for LV lead implantation during cardiac resynchronization therapy (CRT). ${ }^{5}$ All the new gated-SPECT software packages now available in the market have validated capabilities for phase analysis in MPI. ${ }^{6}$ The clinical results of the technique are so significant that Port wrote in this journal few years ago: "Cardiac dyssynchrony: We have the tools. It is time to use them." ${ }^{, 7}$ Why the adoption of phase analysis from gated-SPECT MPI is not universalized in the clinical practice today? First, evidence is still mounting that phase analysis parameters can be used in clinical decision-making. ${ }^{8,9}$ Recently, two studies have shown opposite results. Peix et al. in a nonrandomized, international, multicenter trial: "Value of intraventricular synchronism assessment by gatedSPECT MPI in the management of heart failure patients submitted to cardiac resynchronization therapy" (IAEA VISION-CRT) with 195 consecutive patients was not able to show that baseline dyssynchrony or on-target lead placement was predictive of better clinical response after CRT implantation. ${ }^{10}$ However, Zou et al. in a prospective, randomized trial with 194 consecutive patients, demonstrated that the use of gated-SPECT imaging to assess $\mathrm{LV}$ latest activation improved the rate of on-target LV lead placement, which increased the CRT efficacy. ${ }^{11}$

The second important issue related to the incorporation of phase analysis in clinical practice is the 


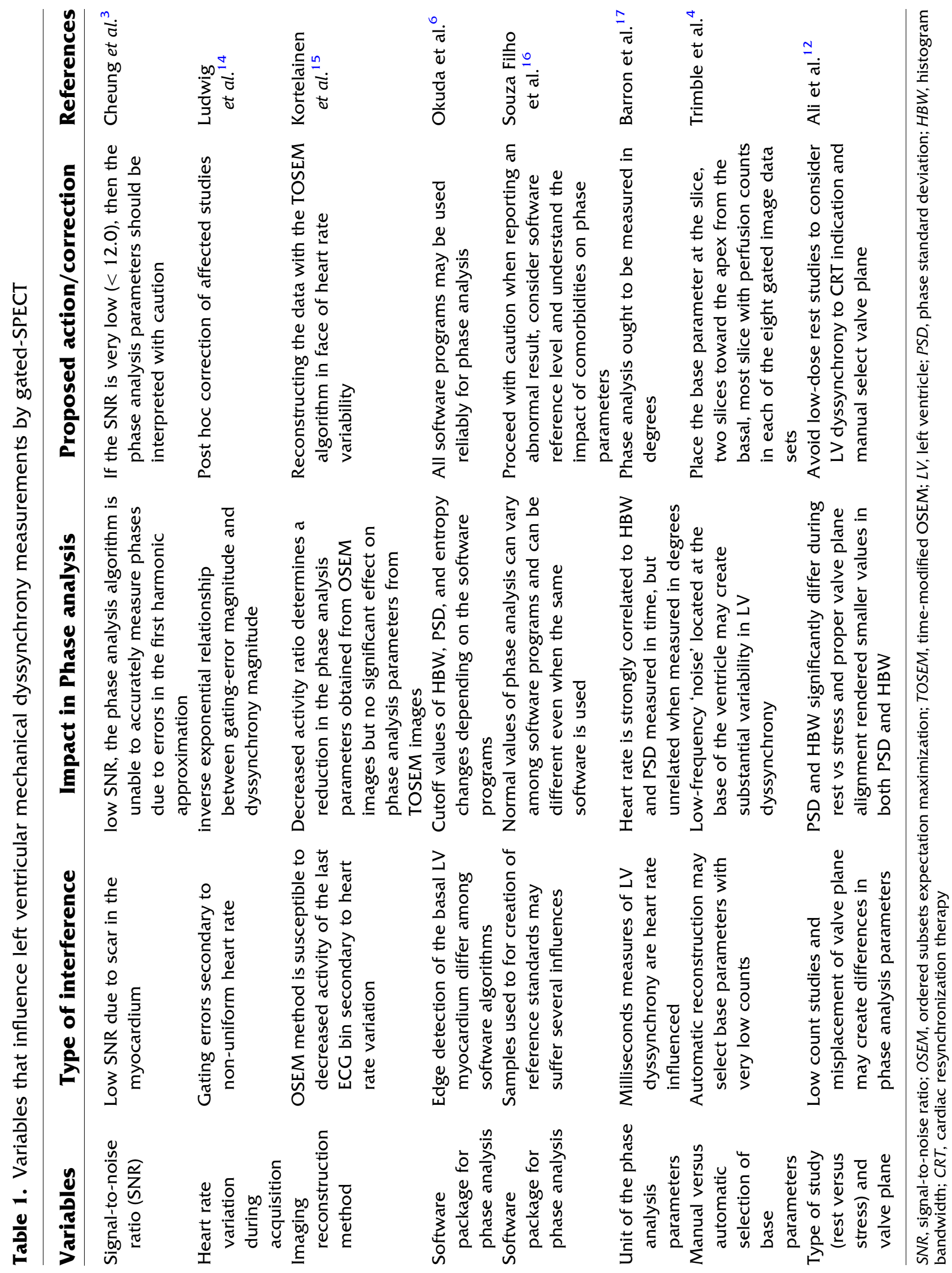


understanding of the clinical, physiological, and physical variables that can influence the results of the technique and how they can compromise its use in the decision-making. In this issue of the Journal, Ali et al, present the results of a retrospective study that examines the impact of the test type and alignment of valve plane in dyssynchrony parameters. ${ }^{12}$ We must congratulate the authors on the extreme detailed approach that they used and the consistency of their results. The mean standard deviation of left ventricular phase (PSD) and phase histogram bandwidth (HBW) during rest were significantly higher compared to that obtained during stress $\left(33.4 \pm 17.4^{\circ} \times 20.7 \pm 13.5^{\circ}\right.$ and $97.7 \pm 59.6^{\circ} \times 59.4 \pm$ $45.4^{\circ}$, respectively). The use rest images could be responsible for falsely labeling $30 \%$ of the patients as having significant LVMD. The most probable explanation to this finding is that stress gated images are associated higher tracer uptake due to hyperemia and consequently more counts which allows more precise dyssynchrony parameters secondary to reduced statistical noise. ${ }^{13}$ Another important observation is that automatic detection of valve plane by algorithm software increased the PDS and HBW significantly compared to the manual base adjustment. The authors pointed out that improper delineation of the valve plane can lead to accounting of the membranous portion of LV septum and part of the atrial myocardium, both having opposite phase from the remainder of the $\mathrm{LV}$, causing imprecise measurements of LV dyssynchrony parameters.

The results of the study of Ali et al. have immediate clinical implications. First, high-quality studies with good myocardial counts should be preferentially used for the evaluation of mechanical dyssynchrony to reduce inaccuracies. Second, the comparison of LV dyssynchrony parameters ought to use the same protocol settings (both stress or rest gated and similar tracer dose) to assure reproducibility. And, finally, the adequate review of the degree of valve plane exclusion and angular alignment is requested to minimize this potential error. The assessment of left ventricular mechanical dyssynchrony using SPECT is critically influenced for some variables (Table 1) that must be known by physicians reporting their results and for the use in the clinical decision-making: signal-to-noise ratio $^{3}$; heart rate variation during acquisition ${ }^{14}$; method of imaging reconstruction ${ }^{15}$; differences in software packages and in their validation ${ }^{6,16}$; unit used for dyssynchrony measurement ${ }^{17}$; manual versus automatic selection of base slice. ${ }^{4}$ Ali et al added new evidence demonstrating that the type of study (rest vs stress) and valve plane are also important in the LV dyssynchrony gated-SPECT results.

Analyzing the recent data and clinical evidence about CRT and phase analysis in gated-SPECT MPI we can perceive the upsides (the good), the downsides (the bad), and the parts that could have been done better, but were not (the ugly). The good: LV dyssynchrony assessed with phase analysis is a robust and mature clinical tool able to generate reproducible and meaningful clinical data that may support and guide clinical decision-making. ${ }^{18}$ The knowledge about the best practices for accurate results is mandatory in using this method. The bad: in spite of more than a decade of clinical research phase analysis is not routinely used in clinical practice as no other reliable imaging assessment of mechanical dyssynchrony is used as prerequisite for the selection of candidates for CRT. ${ }^{19}$ The ugly: despite of more than two decades of clinical use, several studies continuously show that $30 \%$ of patients remain as nonresponders to the CRT. ${ }^{10}$ This gap represents a very significant unmet clinical need and must be carefully addressed in future studies in order to reduce the costs, the morbidity, the mortality, and futility of using an expensive technology, like CRT, in patients that will not derive any benefit. In this context, machine learning can bring valuable contributions. As an example, it is worth highlighting the work of Tokodi et al. who were successful using Random Forests in predicting 1-, 2-, 3-, 4-, and 5-year all-cause mortality from pre-implant variables of patients submitted to CRT. ${ }^{20}$

\section{Disclosure}

Claudio Tinoco Mesquita received travel grant and participated in advisory board for Pfizer in 2019. Principal Investigator for the ongoing Apollo B trial from Alnylam. Received fees for educational presentations for Bayer in 2017. Erito Marques de Souza Filho has no conflict of interest.

\section{References}

1. Safi S, Thiessen T, Schmailzl KJG. Acceptance and resistance of new digital technologies in medicine: Qualitative study. J Med Internet Res 2018;20:1-9.

2. Chen J, Garcia EV, Folks RD, et al. Onset of left ventricular mechanical contraction as determined by phase analysis of ECGgated myocardial perfusion SPECT imaging: Development of a diagnostic tool for assessment of cardiac mechanical dyssynchrony. J Nucl Cardiol 2005;12:687-95.

3. Cheung A, Zhou Y, Faber TL, Garcia EV, Zhu L, Chen J. The performance of phase analysis of gated SPECT myocardial perfusion imaging in the presence of perfusion defects: A simulation study. J Nucl Cardiol 2012;19:500-6.

4. Trimble MA, Velazquez EJ, Adams GL, et al. Repeatability and reproducibility of phase analysis of gated single-photon emission computed tomography myocardial perfusion imaging used to quantify cardiac dyssynchrony. Nucl Med Commun 2008;29:37481.

5. Reis CCW, Nascimento EA, Dias FBR, et al. Applicability of myocardial perfusion scintigraphy in the evaluation of cardiac 
synchronization. Arq Bras Cardiol Imagem Cardiovasc 2017;30:54-63.

6. Okuda K, Nakajima K, Matsuo S, et al. Comparison of diagnostic performance of four software packages for phase dyssynchrony analysis in gated myocardial perfusion SPECT. EJNMMI Res 2017;7:1-9.

7. Port S. Cardiac dyssynchrony: We have the tools. It is time to use them. J Nucl Cardiol 2012;19:420-3.

8. Boogers MM, Van Kriekinge SD, Henneman MM, et al. Quantitative gated SPECT-derived phase analysis on gated myocardial perfusion SPECT detects left ventricular dyssynchrony and predicts response to cardiac resynchronization therapy. J Nucl Med 2009;50:718-25.

9. Henneman MM, Chen J, Ypenburg C, et al. Phase analysis of gated myocardial perfusion single-photon emission computed tomography compared with tissue doppler imaging for the assessment of left ventricular dyssynchrony. J Am Coll Cardiol 2007;49:1708-14.

10. Peix A, Karthikeyan G, Massardo T, et al. Value of intraventricular dyssynchrony assessment by gated-SPECT myocardial perfusion imaging in the management of heart failure patients undergoing cardiac resynchronization therapy. J Nucl Cardiol. 2019. https://doi.org/10.1007/s12350-018-01589-5.

11. Zou J, Hua W, Su Y, et al. SPECT-guided LV lead placement for incremental CRT efficacy: Validated by a prospective, randomized, controlled study. JACC Cardiovasc Imaging 2019;12:2580-3.

12. Ali O, Shenoy M, Alani A, et al. Are rest and stress SPECT MPI measures of dyssynchrony dyssynchronous? J Nucl Cardiol 2020;27:1-10.

13. AlJaroudi W, Alraies MC, DiFilippo F, et al. Effect of stress testing on left ventricular mechanical synchrony by phase analysis of gated positron emission tomography in patients with normal myocardial perfusion. Eur J Nucl Med Mol Imaging 2012;39:66572.

14. Ludwig DR, Friehling M, Schwartzman D, et al. On the importance of image gating for the assay of left ventricular mechanical dyssynchrony using SPECT. J Nucl Med 2012;53:1892-6.

15. Kortelainen MJ, Koivumäki TM, Vauhkonen MJ, Hakulinen MA. Time-modified OSEM algorithm for more robust assessment of left ventricular dyssynchrony with phase analysis in ECG-gated myocardial perfusion SPECT. EJNMMI Phys 2019;6:1-12.

16. Souza Filho EM, Mesquita CT, Gismondi RA, et al. Are there normal values of phase analysis parameters for left ventricular dyssynchrony in patients with no structural cardiomyopathy?: a systematic review. Nucl Med Commun 2019;40:980-5.

17. Barron AJ, Xavier R, Al-Housni M, Reyes E, Underwood R. Phase analysis, a novel SPECT technique for left ventricular dyssynchrony: Are degrees and milliseconds interchangeable? J Nucl Cardiol. 2019. https://doi.org/10.1007/s12350-018-01574-y.

18. Peix A, Mesquita CT, Paez D, et al. Nuclear medicine in the management of patients with heart failure: guidance from an expert panel of the International Atomic Energy Agency (IAEA). Nucl Med Commun 2014;35:818-23.

19. Normand C, Linde C, Singh J, Dickstein K. Indications for Cardiac Resynchronization Therapy: A Comparison of the Major International Guidelines. JACC Hear Fail 2018;6:308-16.

20. Tokodi M, Schwertner WR, Kovács A, et al. Machine learningbased mortality prediction of patients undergoing cardiac resynchronization therapy: the SEMMELWEIS-CRT score. Eur Heart J. 2020. https://doi.org/10.1093/eurheartj/ehz902.

Publisher's Note Springer Nature remains neutral with regard to jurisdictional claims in published maps and institutional affiliations. 